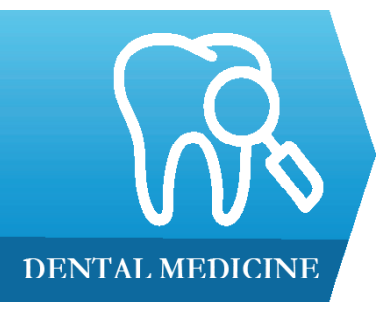

1) Department of Periodontics, RUHS College of Dental Sciences, Jaipur, India

2) Private Dental Practice, Jaipur, India

3) Department of Periodontics, Mahatma Gandhi College of Dental Sciences, Jaipur, India

DOI: $10.15386 / \mathrm{mpr}-1492$

Manuscript received: 19.10.2019

Received in revised form: 22.02 .2020

Accepted: 19.03.2020

Address for correspondence:

dr.varunsaini@gmail.com

This work is licensed under a Creative Commons Attribution-NonCommercialNoDerivatives 4.0 International License

\title{
Effect of commercially available nano-hydroxy apatite containing desensitizing mouthwash on dentinal tubular occlusion: an in vitro FESEM analysis
}

\author{
Neha Saini ${ }^{1}$, Setu Mathur ${ }^{1}$, Varun Saini ${ }^{2}$, Anjali Kapoor ${ }^{1}$, \\ Sharmistha Vijay ${ }^{1}$, Sonia Gurjar ${ }^{3}$
}

\begin{abstract}
Background and aim. Dentinal hypersensitivity is a significant clinical problem encountered in daily dental practice. The management of this condition requires a good understanding of the complexity of the problem, as well as the variety of treatments currently available. The treatment approaches can be either home care products or professionally applied desensitizing agents. The present in-vitro study was designed to investigate the dentinal tubule occluding ability of commercially available nano HA containing mouthwash using FESEM analysis.

Methods. In the present in vitro study, 15 human premolars and canines were taken and sectioned mesiodistally. A total of 30 dentinal samples were obtained. All the dentinal discs were etched with $6 \%$ citric acid for 2 minutes. The treated samples were washed thoroughly with distilled water for 30 seconds. Samples were divided in two groups of 15 each. The specimens in Group I were shaken vigorously in the Vitis Sensitive mouthwash for 2 min twice daily for 14 days. After this intervention samples were placed in distilled water. Group II specimens were immersed in distilled water. Samples were subjected to FESEM to analyze for tubular occlusion.

Results. In group I nearly complete dentinal surface occlusion was present on the $7^{\text {th }}$ and $14^{\text {th }}$ day and precipitates were seen covering a large part of the dentinal surface. In group II no dentinal tubular occlusion was observed.

Conclusion. The results of the present study support the ability of nHA containing Vitis sensitive mouthwash to occlude the dentinal tubules and thus it may demonstrate a significant reduction in dentinal hypersensitivity when used clinically.
\end{abstract}

Keywords: dentine hypersensitivity, mounthwash, scanning electron microscope

\section{Background and aim}

Dentinal hypersensitivity (DH) is defined as "short, sharp pain arising from exposed dentine in response to stimuli, typically thermal, evaporative, tactile, osmotic or chemical and which cannot be ascribed to any other dental defect or pathology" as per international workshop on DH $[1,2]$.

It is a clinical condition that affects around $4-74 \%$ of the population, depending on diagnostic criteria, selection method or time frames examined. It is more prevalent in the age group of 20-50 yrs but it may affect patients of any age [3]. DH negatively affects the lives of the people as they become selective in eating habits because of discomfort and pain on consuming hot and cold food items and may become non-compliant in maintaining daily oral hygiene which may lead to a vicious cycle of poor oral hygiene and DH. Perception of pain may vary from slight discomfort to severely impaired quality of life.

DH primarily develops in two phases, namely: lesion localisation and lesion initiation. Studies suggest that 
lesion localization occurs by loss of protective enamel covering over the dentin. Enamel can be lost through attrition, toothbrush abrasion, erosion, and abfraction, gingival recession, pocket reduction, tooth preparation for crown or secondary to periodontal diseases. It is acknowledged that for DH to occur, the lesion localization has to be initiated $[4,5]$.

\section{Theories and histopathogenesis of DH}

The pathogenesis of any disease should be understood well in order to conduct its proper management and treatment. Three major theories have been proposed regarding the mechanisms of dentin hypersensitivity. The direct innervation theory states that nerve endings extend through the pulp and dentin up to the dentinoenamel junction. The odontoblast receptor theory suggests that odontoblasts act as receptors and are responsible for relaying a signal to a nerve terminal. These two theories have various limitations which has given rise to a newer concept that sensitivity develops from fluid movement within the dentinal tubules stimulating mechanoreceptors on the pulpal nerves - the hydrodynamic theory proposed by Gysi year and explained further by Brannstrom, 1981 $[3,6,7]$.

An increased outward fluid flow causes a pressure change across the dentine, distorting the A- $\delta$ fibers by a mechanoreceptor action, causing sharp, and shooting pain.

Notably, evidence from Scanning Electron Microscope (SEM) studies completed until now have shown that hypersensitive dentin has a large number of wide, open dentinal tubules on the surface rather than nonsensitive dentin $[8,9]$. Around 30,000 tubuli per $\mathrm{mm}^{2}$ of 1-2 $\mu \mathrm{m}$ diameter run perpendicularly from the dentin surface to the dental pulp [10].

\section{Management and treatment of DH}

Management of DH is aimed to alleviate the pain and concern of the patient and to treat the underlying dental defect, if any. Pashley in 1986 suggested that DH might be reduced physiologically by the formation of intratubular crystals from dentinal fluids and saliva minerals [6].

Therefore, if the principles of hydrodynamics are acknowledged, two treatment approaches for DH appear to reduce or potentially eliminate hypersensitivity. Firstly those that result in the physical occlusion of tubules by substance which blocks the diffusion through the tubules into the dentin sub-surface eg. Strontium salt, fluoride, potassium oxalate, hydroxyapatite, nano hydroxyapatite etc. and secondly those that block neural transmission at the pulpal tissues, chemically depolarizing the nerve synapse eg. silver nitrate, zinc chloride, potassium salts etc [11].

Recently LASER has been used for the treatment of DH. The proposed mechanisms for laser and its effect on reducing DH include:

1. Occlusion through coagulation of the proteins of the fluid inside the dentinal tubules

2. Occlusion of tubules through partial sub-melting

3. Discharging of internal tubular nerve

More recently, the adhesive resins system has appeared which includes varnishes, bonding agents, and repairing resin composites which can effectively seal dentinal tubules by forming a hybrid layer. These new adhesives, act in a way that the smear layer will be modified and incorporated into the hybrid layer. It is claimed that the recent dentin bonding agents can prevent DH. For example, Gluma desensitizing agent (Heraeus Kulzer; $\mathrm{Ca}$, USA) includes hydroxyethyl methacrylate (HEMA), benzalkonium chloride, gluteraldehyde and fluoride. Gluteraldehyde can lead to protein coagulation within dentinal tubules. Casein-phosphopeptide-amorphous calcium phosphate (CPP)-(ACP) under the name GC Tooth Mousse is a remineralizing agent and is claimed to be effective in the prevention and treatment of DH [12].

The choice of the desensitizing agent delivery vehicle should be in accordance with the Grossman criteria for an ideal desensitizing agent, i.e. with long-term effects, nonirritant to pulp, painless, and easy to apply and should not stain the tooth [13].

From the scientific literature available currently, it is evident that there is no recognized ideal desensitizing product that provides both fast acting and long lasting protection against the pain associated with DH. This concern has led to the development of novel substances such as Bioactive glasses, hydroxyapatite (HA), nano HA, nanocarbonate apatite crystal and potassium oxalate oral rinses etc [14].

The treatment approaches can be either home care products i.e. toothpastes, gels, mouthwashes or professionally applied desensitizing agents i.e. varnish etc. Toothpastes are the most preferred ones since they are simple to use, inexpensive and readily available as an over the counter product $[3,15]$. The primary agent for at-home desensitizing toothpastes is potassium which disturbs the transmission of nerve endings. Potassium salts, including potassium nitrate, potassium chloride or potassium citrate act by blocking the neural transmission at the nerve synapse by diffusing along the dentinal tubules and depolarizing the nerve cells. The effects of potassium salts are cumulative and it takes several weeks to exert its desensitising action. Fluoride has been the principal component in remineralisation. It has an important role in the prevention and treatment of dental caries and has now become an affirmative desensitising agent [16].

The recent trend in biomaterials research is focused on nanotechnology. The search for an agent that would predictably and permanently occlude the tubules and blend with them has prompted the use of HA which is the principal constituent of teeth. Hydroxyapatite is used in 
toothpaste for the re-mineralization of enamel. With their high area to volume ratio, nano-sized HA particles are expected to be excellent materials for these applications.

Nano-HA is a bioactive and biocompatible material with wide applications in both medicine and dentistry. It acts by occluding the dentinal tubules and limiting the movement of the dentinal fluid [17]. Li et al. stated that having a particle size of $20 \mathrm{~nm}$, nano-HA resembles the natural building blocks of enamel and is now affirmatively used as an anti-caries agent, repairing and occluding the dentinal tubules $[18,19]$. The surface density of dentinal tubules of hypersensitive teeth is higher and larger compared to non-sensitive teeth [20]. Its nano-sized particles have a similarity to the apatite crystals of tooth enamel in morphology and crystal structure. Also, they have a capacity of hydration and wettability which allow them to release calcium and phosphate to teeth in adequate concentration [21]. Nano HA toothpaste and oral rinses thus have been developed for home use and have shown to be an attractive alternate to the traditional products.

A few of these agents have also been evaluated as a mouthwash with favorable results, although the evidence supporting their efficacy is low and nonconclusive. However, no hypersensitivity treatment was shown to be fully effective for all patients.

Therefore, given these results, the present study aimed to investigate the ability of a new commercially available nano HA containing desensitizing mouthwash ( Vitis, Dentaid SL Spain) to occlude dentinal tubules in an in -vitro setup.

The Vitis Sensitive mouthwash contains potassium nitrate, xylitol, PVP, sodium hexametaphosphate, $0.172 \%$ sodium monoflourophosphate, provitamin B5, allantoin, $0.0125 \%$ nanohydroxyapatite and potassium chloride.

\section{Methods}

The present study was conducted in the Department of Periodontics, Rajasthan University of Health SciencesCollege of Dental Sciences, Jaipur, Rajasthan, India in the month of January - February 2019. In this in vitro study, 15 non carious extracted human maxillary and mandibular premolars and canines were taken and stored in a buffer solution. Teeth were disinfected with 20,000 parts per million (ppm) of sodium hypochlorite for 24 hours at room temperature. Premolars and canines were taken since they are the most affected by dentinal hypersensitivity. The buccal aspect of the cervical area is the most commonly affected [22]. The teeth were sectioned mesiodistally using a diamond disc to obtain two samples per tooth. A total of 30 dentinal samples $1 \mathrm{~mm}$ thick were obtained. All the dentinal discs were etched with $6 \%$ citric acid for 2 minutes to facilitate the removal of the smear layer and to dis-occlude dentinal tubules. These treated samples were washed thoroughly with distilled water for $30 \mathrm{sec}$ to remove any remaining acid or debris present.

The specimens were then randomly assigned to:

Group I (Vitis mouthwash) - 15 samples

Group II (Distilled water) - 15 samples.

The specimens in Group I were shaken vigorously in the mouthwash for 2 min twice daily for 15days. After intervention samples were placed in distilled water. Group II were immersed in distilled water and received no intervention. Samples were subjected to:

(1) Field Emission Scanning Electron Microscopy (FESEM) (Nova Nano FESEM, model 450, FEI company, Hillsboro, Oregon, America) analysis for tubular occlusion.

The total number of tubules, open tubules, completely occluded tubules and partially occluded tubules were calculated in each image of all of the samples.

(2) Energy dispersive X Ray spectroscopy (EDX) analysis for detection of elemental composition at baseline, $7^{\text {th }}$ day and $14^{\text {th }}$ day. All the images were captured at the same pixel resolution .

Evaluation of the chemical elements present in the dentin disks was done using the EDX technique and analyses of samples previously evaluated by FESEM were performed in order to determine the chemical elements present in the dentinal tubules after the application of desensitizing mouthwash.

Evaluation of the Tubule Occlusion by FESEM.

The sample disks were rinsed with distilled water then dried. The samples were sputter coated with an extremely thin layer $(1.5-3 \mathrm{~nm})$ of gold. This makes the dentine disks conductive to the current. Then samples were subjected to FESEM analysis with a beam voltage of $15 \mathrm{kV}$. The samples were photographed at $5000 \mathrm{X}$ and 10000X magnification and images of 1536* 1103 pixels were obtained. The images were not enhanced and no transformation was carried out.

The ranking criterion was based on the assessment of surface characteristics and patency or occlusion of the dentinal tubules as seen on the FESEM photomicrograph. The standardized FESEM microphotographs were analysed by two blind evaluators according to the scoring criteria previously described in the literature [23]. Two blinded evaluators were trained and calibrated for the evaluation of dentinal occlusion.

Type 0: open dentinal tubules

Type 1: partial dentinal tubule occlusion $<25 \%$ of dentinal tubule orifice.

Type 2: partial dentinal tubule occlusion $>25 \%$ upto $75 \%$ of dentinal tubule orifice.

Type 3: nearly complete dentinal tubule occlusion of $>75 \%$ of dentinal tubule orifice.

At the time of analysis, Type 1 and Type 2 were considered in the same category as the partial dentinal tubule occlusion.

The percentage of occluded dentinal tubules was calculated as described by Jena et al. 2017; dividing the 
total number of occluded dentinal tubules by the total number of dentinal tubules in photomicrograph [24]. The result thus obtained was then multiplied by 100 to obtain the percentage of occluded dentinal tubules for each photomicrograph. The calculations were performed by two blind investigators and the average of the two was taken and tabulated.

The FESEM analysis was done on the dentinal disks and the areas were selected by two blind evaluators on a random basis and the average of them was tabulated.

Evaluation of elemental composition by EDX analysis. EDX analysis was made to qualitatively determine the elemental composition of the precipitates found next to the dentinal tubule.

\section{Statistical analysis}

Data obtained was entered into the MS excel sheet. To assess the reliability of obtained results from the FESEM analysis, detailed statistical analysis was also carried out using the Statistical Package for the Social Sciences (SPSS version 10.5) (IBM Corporation, Armonk, New York, United States). Student's Paired t test was used to assess the significant difference between the groups. The $\mathrm{p}$ value was considered as significant when less than 0.05 .

\section{Results \\ Group I}

Figure 1, 2 shows the FESEM image at baseline, all dentinal tubules are open similar to hypersensitive dentin, indicating that the smear layer produced during the cutting process was removed and the tubules were cleansed as a result of the citric acid treatment. The dentin tubules were completely free from debris.

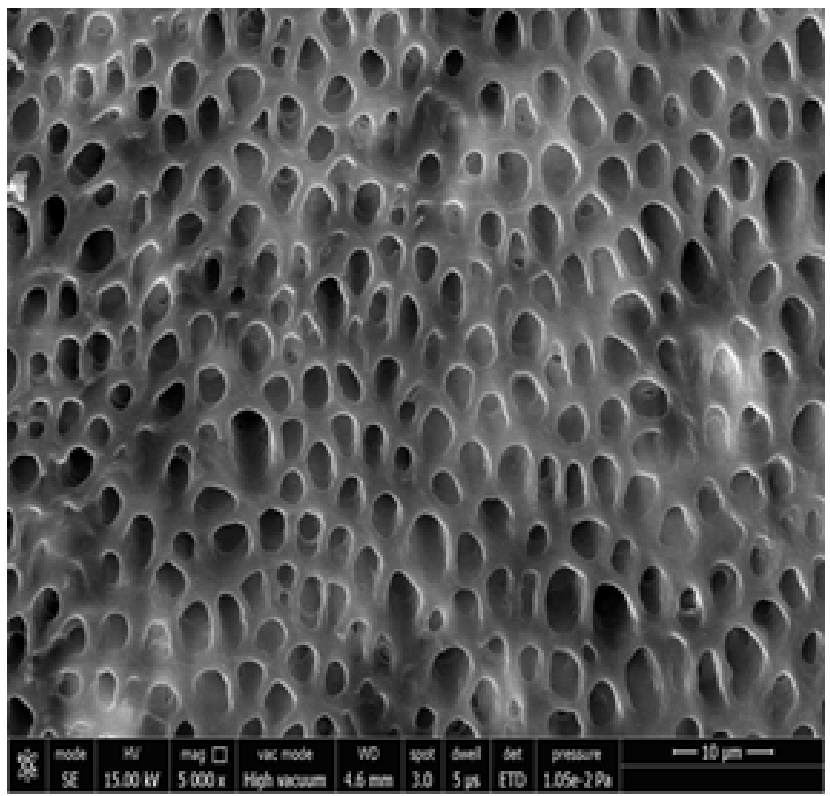

Figure 1. Photomicrograph showing open dentinal tubules after acid etching at 5000x.

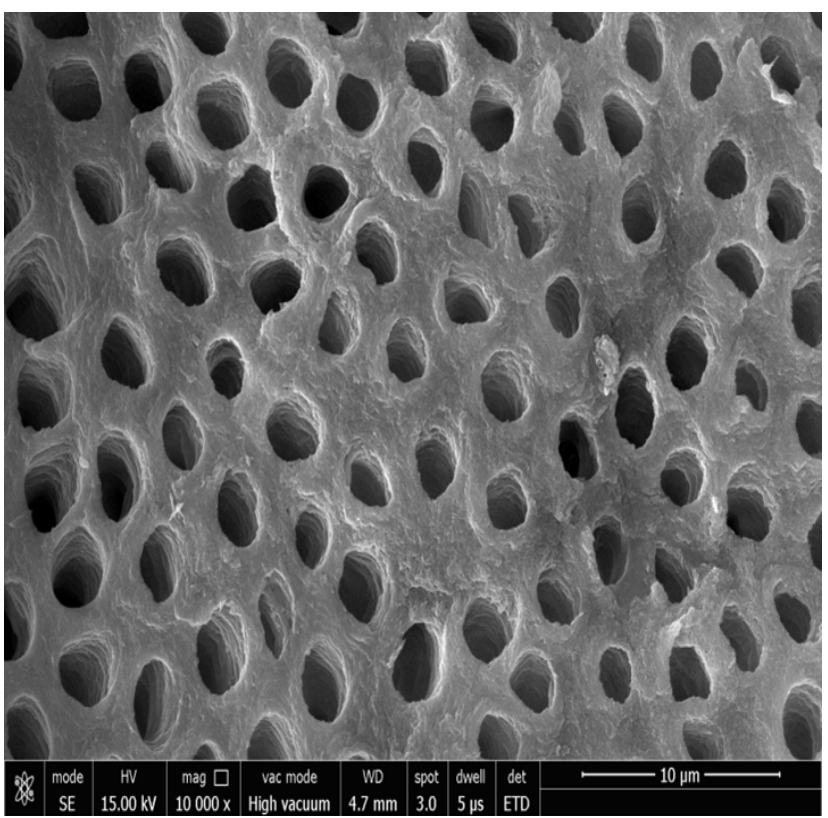

Figure 2. Photomicrograph showing open dentinal tubules after acid etching at 10000x.

Figure 3, 4 shows the FESEM image on the $7^{\text {th }}$ day; a partial occlusion of the dentine tubules by the nHA particles can be seen. When the disks were treated with nHA mouth wash for 2 minutes twice daily for a week, a number of dentinal tubules were seen to be occluded. The nHA particles appear to cover the dentine surface as well as penetrate into the dentine tubules. Peritubular, intertubular and some smear layer can be seen. There was a statistically significant difference in the tubular occlusion between the groups at the end of $7^{\text {th }}$ day $(\mathrm{p}<0.001)$.

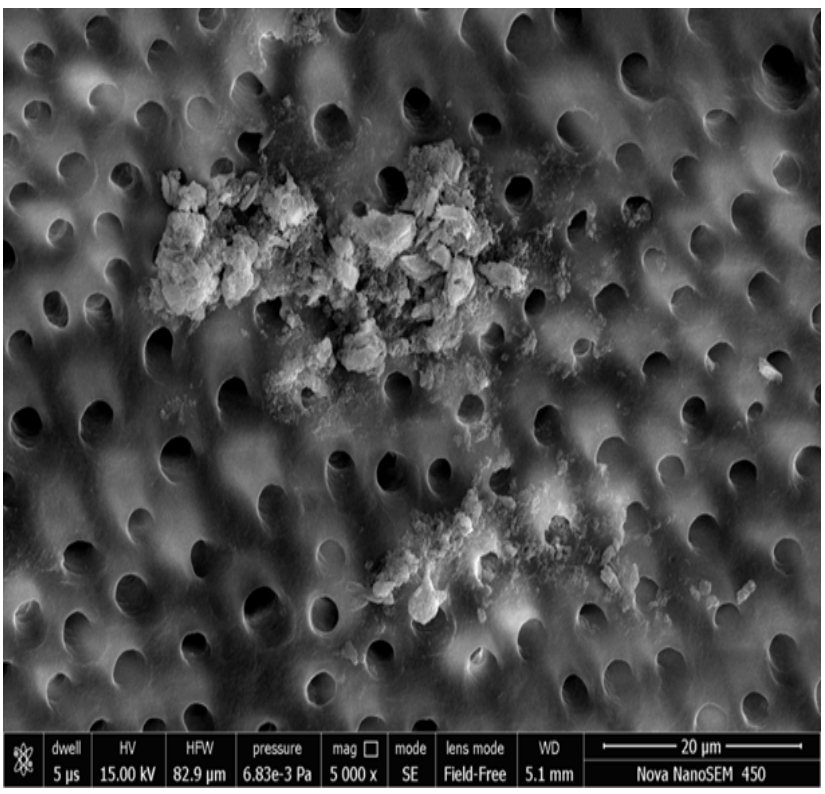

Figure 3. Group I Photomicrograph showing partially occluded dentinal tubules on day 7 at $5000 x$. 


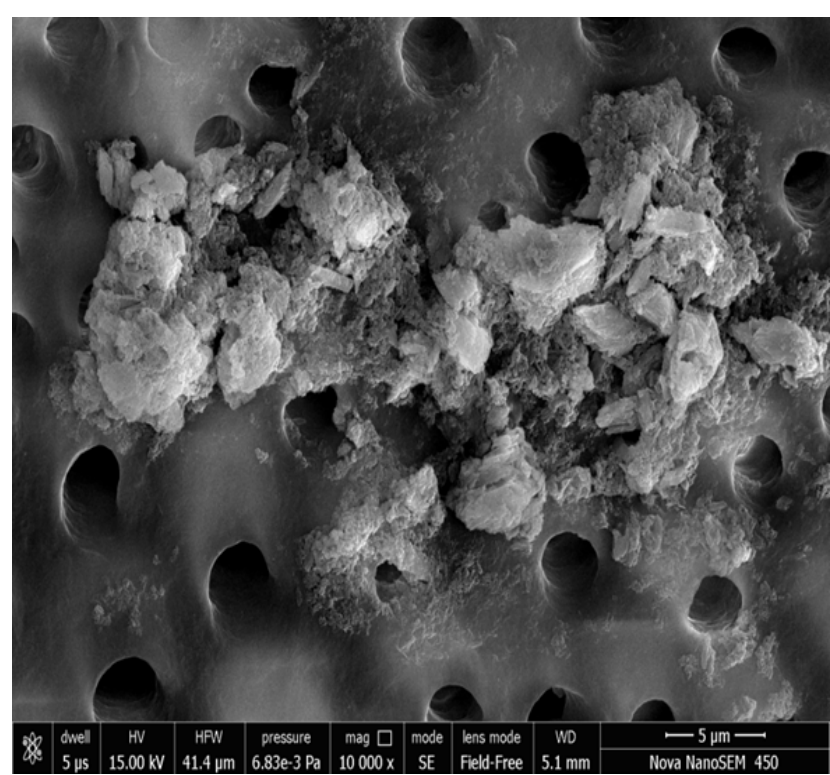

Figure 4. Group I Photomicrograph showing partially occluded dentinal tubules on day 7 at $10000 x$.

Figure 5, 6 show the FESEM image on the14th day; a nearly complete dentinal surface occlusion can be visualized and precipitates were seen covering a large part of the dentinal surface. Peritubular, intertubular and some smear layers can be visualized. Dentinal tubules were occluded from the dentin surface to a depth of approximately $5 \mu \mathrm{m}$ (Figure 7). In addition, precipitates were observed to have a better sealing effect in parts of dentinal tubules, reaching a depth of approximately $20 \mu \mathrm{m}$ (Figure 8). There was a statistically significant difference in the tubular occlusion between the groups at the end of $14^{\text {th }}$ day $(\mathrm{p}<0.001)$.

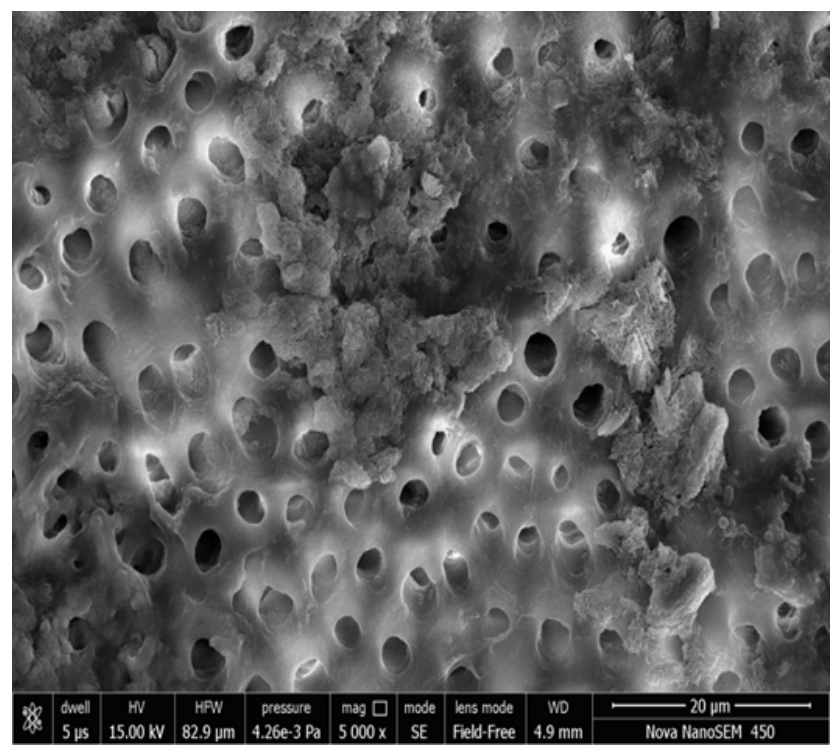

Figure 5. Group I Photomicrograph showing nearly complete occluded dentinal tubules on day 14 at 5000x.



Figure 6. Group I Photomicrograph showing nearly complete occluded dentinal tubules on day 14 at 10000x.

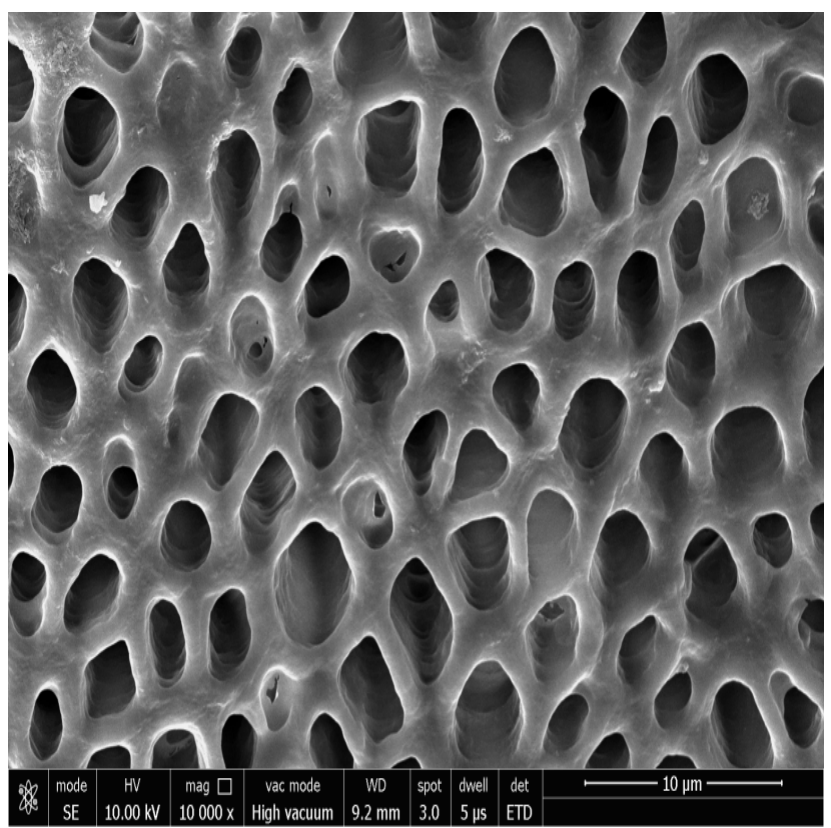

Figure 7. Group II Photomicrograph showing open dentinal tubules on day 14 at $10000 x$.

\section{Group: 2}

Figure 7 shows the FESEM image in Control Group. All the dentinal tubules were seen to be opened.

\section{EDX ANALYSIS}

EDX analysis provided qualitative information of the presence of calcium $(\mathrm{Ca})$, phosphorous $(\mathrm{P})$, potassium $(\mathrm{K})$ and oxygen $(\mathrm{O})$ on the $14^{\text {th }}$ day (Figure 8$)$. It revealed the peaks of the chemical elements. High peaks of calcium and phosphorous were observed in Group 1. 


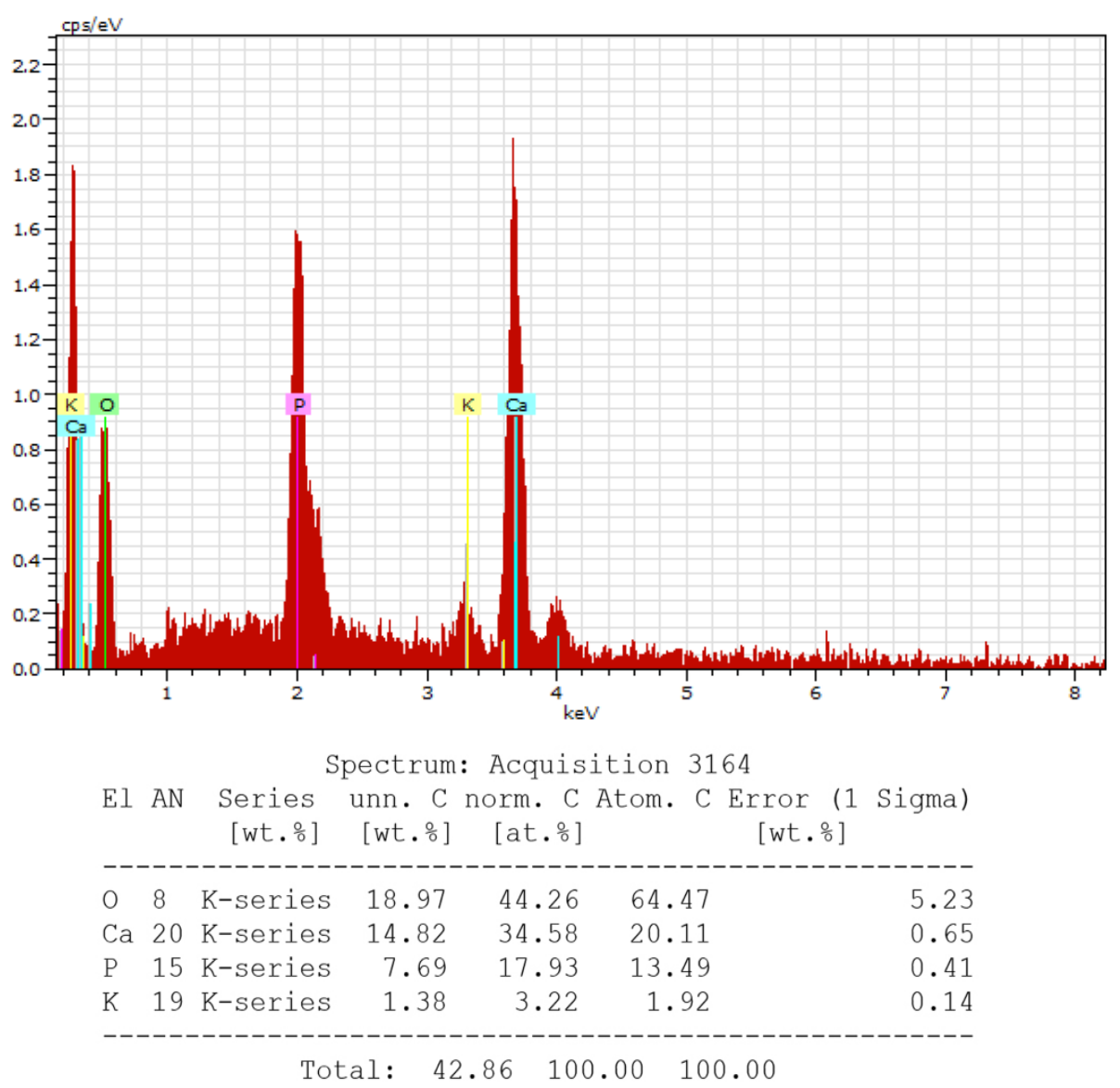

Figure 8. EDX analysis showing presence of calcium $(\mathrm{Ca})$, phosphorous $(\mathrm{P})$, potassium $(\mathrm{K})$ and oxygen $(\mathrm{O})$ at $14^{\text {th }}$ day.

Table I. Comparison of level of occlusion of dentinal tubules between the two groups at baseline and post-treatment time intervals using Student's paired t-test.

\begin{tabular}{|c|c|c|c|c|c|c|c|}
\hline \multirow[b]{2}{*}{ Level of Occlusion } & \multicolumn{3}{|c|}{ Group 1} & \multicolumn{3}{|c|}{ Group 2} & \multirow[b]{2}{*}{ p-value } \\
\hline & At baseline & $\underset{7^{\text {th }} \text { day }}{\text { dat }}$ & $\begin{array}{c}\text { At } \\
14^{\text {th }} \text { day }\end{array}$ & $\begin{array}{c}\text { At } \\
\text { baseline }\end{array}$ & $\underset{7^{\text {th }} \text { day }}{\text { dat }}$ & $\underset{14^{\text {th }} \text { day }}{\text { dat }}$ & \\
\hline Open dentinal tubules & $100 \%$ & $0 \%$ & $0 \%$ & $100 \%$ & $100 \%$ & $100 \%$ & \multirow{3}{*}{$\begin{array}{l}<0.001 \\
\text { Statistically } \\
\text { significant }\end{array}$} \\
\hline Partial dentinal tubules & $0 \%$ & $70 \%$ & $25 \%$ & $0 \%$ & $0 \%$ & $0 \%$ & \\
\hline Nearly complete dentinal tubules & $0 \%$ & $30 \%$ & $75 \%$ & $0 \%$ & $0 \%$ & $0 \%$ & \\
\hline
\end{tabular}

\section{Discussion}

The present in-vitro study was designed to investigate the dentinal tubule occluding ability of commercially available nano HA containing mouthwash using FESEM analysis.

The challenges of reducing $\mathrm{DH}$ has attracted considerable interest because of the rapid increase in its prevalence rate ranging from $5 \%$ to $85 \%$ [9] and $2-8 \%$ to $74 \%$ [22]. There are various approaches for reducing the excitability of nerve fibres such as the application of antiinflammatory agents, desensitizing agents, occlusion of dentinal tubules as well as root coverage by periodontal surgery [25,26]. Grossman proposed that an ideal desensitizing agent should be rapidly acting, have a longterm effect, be harmless to pulp, painless, easy to apply, not stain the teeth and be consistently effective. An ideal desensitizing agent as proposed by Grossman in 1935 is yet to be identified [13].

Vitis Desensitising mouthwash has a dual action for reducing the DH. It contains potassium nitrate which reduces the excitability of nerve fibres by chemical action and nano HA which either physically occlude the 
dentinal tubules or reduces the diameter and thus limits the movement of dentinal fluids. Being a natural component of tooth and bone, nano particles of HA ensure a complete integration with the tooth enamel by forming a stable bond with the HA of teeth [26].

According to the manufacturer, the effectiveness of the mouthwash is based on its ability to provide ions of calcium, phosphate, and fluoride to demineralized tooth surfaces, which can be reorganized in the form of hydroxyapatite, fluorapatite, and calcium fluoride, with acid resistance similar to that of the natural tooth.

In this in vitro study, 15 non carious extracted human maxillary and mandibular premolars and canines were taken and sectioned mesiodistally to obtain two samples per tooth. A total of 30 dentinal samples were obtained. Premolars and canines were used since they are the most affected by dentinal hypersensitivity.

The dentin samples were subjected to $6 \%$ citric acid for 2 minutes; this removed the smear layer and made the dentinal tubules funnel shaped to enable the particles of desensitizing mouthwash to pass through (Fig 1,2). The results of the present study are in agreement with studies completed by Mahajan Neha and Vandana KL (2015) [21].

The most effective variable for affecting fluid flow in dentine is the radius of the tubule. The fluid flow through the dentinal tubule is inversely proportional to the fourth power of the radius. Thus, doubling the tubule diameter, results in a 16 fold increase in the fluid flow, thereby increasing hypersensitivity. This explains the concept of why tubular occlusion, of whatever nature, is thought to reduce the pain of dentin hypersensitivity. Based on this theory, $6 \%$ citric acid is used in in vitro studies to simulate DH. Therefore, it leads to acid etching of the dentinal surface resulting in a wider dentinal tubular surface.

The vigorous shaking of the disks was performed in both groups to mimic the clinical conditions during oral rinsing. The disks were then placed in distilled water.

On the $7^{\text {th }} \& 14^{\text {th }}$ day the disks were subjected to FESEM analysis for quantitative and qualitative determination of tubular occlusion. Prior to this, the dentinal disks were sputter coated which made them conductive. The tooth being nonconductive, the surface of disks behaves as an electron trap. These electrons accumulate on the surface and create an extra white region that interferes with the image formation which is called charging. Sputter coating is a physical vapor deposition of a thin film of conducting material such as gold, silver, platinum, chromium etc. This thin film removes the electrons from the disks; increases signal to noise ratio and improves the image quality and resolution during imaging [27].

In the present study, on the 14th day, a nearly complete dentinal surface occlusion can be visualized and precipitates were seen covering a large part of the dentinal surface. Peritubular, intertubular and some smear layers were also seen. The better tubule occluding ability of
nHA can be attributed to its nano particle size. This gives nHA higher functional properties such as high bioactivity, osteoconductivity and crystallanity. There is an increase in the surface area and better ingress of the ions into tubules leading to better occlusion [28].

Two main approaches have been suggested towards remineralizing the demineralized dentin. It takes place by either heterogenous nucleation or biomineralisation mechanism. In this study, vigorous shaking of the dentinal disc samples in n-HA mouthwash twice daily led to the deposition of n-HA crystals. The results of the present study are in accordance with the studies completed by Zhang S. (2003) which states the formation of material assembly up from the nanoscopic scale, such as molecules and atoms to form larger structures [29]. This is called a bottom up approach which assists in the biomineralisation of dentinal tubules \& its occlusion. [30]. The $\mathrm{Ca}++$ and PO4- ions form a super saturated solution in which the nano HA crystals precipitate to form a mineralized matrix [31].

The efficacy of treatment is established using FESEM analysis of high magnification $(5000 \mathrm{X}$ and $10,000 \mathrm{X})$. The results from the FESEM analysis of the test and control mouthwashes provided a wide variation in the manner and extent of the surface precipitate. There was a significant occlusion of the dentinal tubules in the group I discs which approximated to $75 \%$ at day 14 . There was no occlusion observed in the dentinal tubules in group II at day 14.

The intergroup comparison performed using Student's paired $t$-test in shown in Table I, which reveals a statistically significant difference in the pretreatment and posttreatment values in group I compared with group II.

The EDX analysis showed the peak levels of calcium \& phosphorous which is consistent with the remineralisation of the dentin surface and occlusion of the dentinal tubules by a dentin like mineral (Figure 8, 9). The peaks of $\mathrm{P}, \mathrm{O}$ and $\mathrm{Ca}$ indicate the presence of the compounds of these elements (calcium hydroxyapatites) which are the components of the tested mouthwash. This shows that the occlusion of dentinal tubules is generated by HA crystal precipitate.

A study by Yuan et al. in 2012 stated that HA containing dentifrice can be effective in $>90 \%$ in plugging the dentinal tubules [32]. Ishikawa et al. 1994 reported that apatite mineral is deposited not only on the dentine surface but also to a depth of 10-15 $\mu \mathrm{m}$ inside the dentinal tubules [33]. KNO3 has also been shown to have a desensitizing effect on dentinal tubules. It acts by nerve desensitization rather than by dentinal tubular occlusion [34]. A study by James and Manjunath 2017 reported the tubule occlusion effect of $\mathrm{KNO} 3$ toothpaste and mouthwash when applied for 4 weeks [35].

Morris et al., observed that in various in vivo studies on DH, a very powerful placebo effect was noted, reasonably since there was a lower number of subjects. 
Additionally, large standard deviations were observed, because the highly subjective nature of pain and variability of the individual pain response in dentin sensitivity studies makes it extremely difficult to detect significant differences between groups without utilizing a large number of subjects [36].

Sodium monofluorophosphate present in the mouthwash has synergistic effects along with nano HA in occluding the dentinal tubules. It acts as a F - and PO4 source for the precipitation of apatite crystals within dentinal tubules and aids in remineralisation as fluoride and phosphate deposits acts as seeds for heterogenous nucleation [37].

According to Wilchgers and Ermert and Kim, the desensitizing effect of potassium nitrate is due to the increase in concentration of extracellular potassium around the nerve fibers which causes their depolarization, avoids repolarization, and blocks the axonic action. This blocks the passage of the nerve stimulus, resulting in inactivation of the action potential. In the present study, potassium nitrate was used as a mouthwash. Mouthwashes usually are administered in lower concentrations compared to dentifrices because of the risk of being swallowed [38].

Another component of the mouthwash was Provitamin B5 which has long been used as a cosmetic agent because of its humectant action. It is in an inactive form but is readily converted in the skin to pantothenic acid. Pantothenic acid is incorporated into the energy cycle of the cell and promotes gingival epithelisation, regeneration and reduction of inflammation [39].

However, the in-vitro studies have some inherent limitations. They were not able to mimic the oral conditions. The dentinal discs after intervention were placed in distilled water, so the effect of saliva on the mineralization could not be noted. Also, the effect of food, toothbrush action $\&$ durability could not be ascertained. Furthermore, the hydraulic conductance measurements were not taken and only the surface precipitation was evaluated. Lastly root dentine taken from the cervical region of the tooth would had been a more appropriate model than mid coronal sections; however, there is a greater variability in terms of tubule orientation of the root dentine compared to mid coronal dentine. The present study has been limited to a two-week application (twice daily) of the desensitizing agents on the prepared dentinal samples. Most of the dentinal tubules are occluded and if the application period was increased, there are chances of occluding a greater number of dentinal tubules.

\section{Conclusion}

Within the limitations, the results of the present study, confirmed by FESEM analysis support the ability of nHA containing Vitis sensitive mouthwash to occlude the dentinal tubules and thus it may demonstrate a significant reduction in dentinal hypersensitivity in clinical studies, if applied for a sufficient period. In a clinical perspective, it appears to be a good candidate and should be further investigated using a validated questionnaire and clinical trials. Future in vivo studies, comparing Vitis sensitive mouthwash to other available desensitizing products, may highlight its efficacy in clinical practices.

\section{Acknowledgement}

We would like to thank staff members of the Malviya National Institute of Technology (MNIT), Jaipur for helping us in the FESEM work.

\section{References}

1. Canadian Advisory Board on Dentin Hypersensitivity. Consensus-based recommendations for the diagnosis and management of dentin hypersensitivity. J Can Dent Assoc. 2003;69:221-226.

2. Gillam DG. Current diagnosis of dentin hypersensitivity in the dental office: an overview. Clin Oral Investig. 2013;17 Suppl 1: S21-S29.

3. Miglani S, Aggarwal V, Ahuja B. Dentin hypersensitivity: Recent trends in management. J Conserv Dent. 2010;13:218224.

4. Sabir M, Alam MN. Milk as Desensitizing Agent for Treatment of Dentine Hypersensitivity Following Periodontal Treatment Procedures. J Clin Diagn Res. 2015;9:ZC22ZC25.

5. Orchardson R, Gillam DG. Managing dentin hypersensitivity. J Am Dent Assoc. 2006;137:990-998; quiz 1028-9.

6. Pashley DH. Dynamics of the pulpo-dentinal complex. Crit Rev Oral Biol Med. 1996; 7:104-133.

7. Braennstroem M, Astroem A. A study on the mechanism of pain elicited from the dentin. J Dent Res. 1964;43:619-625.

8. Absi EG, Addy M, Adams D. Dentine hypersensitivity. A study of the patency of dentinal tubules in sensitive and nonsensitive cervical dentine. J Clin Periodontol. 1987;14:280284.

9. Gopinath NM, John J, Nagappan N, Prabhu S, Kumar ES. Evaluation of Dentifrice Containing Nano-hydroxyapatite for Dentinal Hypersensitivity: A Randomized Controlled Trial. J Int Oral Health. 2015;7:118-122.

10. Hiller KA, Buchalla W, Grillmeier I, Neubauer C, Schmalz $\mathrm{G}$. In vitro effects of hydroxyapatite containing toothpastes on dentin permeability after multiple applications and ageing. Sci Rep. 2018;8:4888.

11. Porto IC, Andrade AK, Montes MA. Diagnosis and treatment of dentinal hypersensitivity. J Oral Sci. 2009;51:323-332.

12. Davari A, Ataei E, Assarzadeh H. Dentin hypersensitivity: etiology, diagnosis and treatment; a literature review. J Dent (Shiraz). 2013;14:136-145.

13. Grossman L. A systematic method for the treatment of hypersensitive dentine. J Am Dent Assoc. 1935; 22:592-8.

14. Hill RG, Chen X, Gillam DG. In Vitro Ability of a Novel 
Nanohydroxyapatite Oral Rinse to Occlude Dentine Tubules. Int J Dent. 2015;2015:153284.

15. Sharda S, Prasad KVV, Shetty PJ, Nikhil K. Effectiveness of Desensitizing Dentifrice and Mouthwash on Dentin Hypersensitivity and Tooth Remineralization. Contemp Clin Dent. 2018;9:415-420.

16. Hong JY, Lim HC, Herr Y. Effects of a mouthwash containing potassium nitrate, sodium fluoride, and cetylpyridinium chloride on dentin hypersensitivity: a randomized, doubleblind, placebo-controlled study. J Periodontal Implant Sci. 2016;46:46-56.

17. Hannig M, Hannig C. Nanomaterials in preventive dentistry. Nat Nanotechnol. 2010;5:565-569.

18. Li X, Wang J, Joiner A, Chang J. The remineralisation of enamel: a review of the literature. J Dent. 2014;42 Suppl $1:$ S12-S20.

19. Hannig C, Hannig M. Natural enamel wear--a physiological source of hydroxylapatite nanoparticles for biofilm management and tooth repair? Med Hypotheses. 2010;74:670-672.

20. Addy M, Mostafa P, Newcombe RG. Dentine hypersensitivity: the distribution of recession, sensitivity and plaque. J Dent. 1987; 15:242-248.

21. Neha M, Vandana KL. Effects of citric acid \& desensitizing agent application on nonflourosed \& flourosed dentin: an in vitro FESEM study. Open Dent J. 2015; 9:98-102.

22. Que K, Guo B, Jia Z, Chen Z, Yang J, Gao P. A crosssectional study: non-carious cervical lesions, cervical dentine hypersensitivity and related risk factors. J Oral Rehabil. 2013;40:24-32.

23. Kunam D, Manimaran S, Sampath V, Sekar M. Evaluation of dentinal tubule occlusion and depth of penetration of nanohydroxyapatite derived from chicken eggshell powder with and without addition of sodium fluoride: An in vitro study. J Conserv Dent. 2016;19:239-244.

24. Jena A, Kala S, Shashirekha G. Comparing the effectiveness of four desensitizing toothpastes on dentinal tubule occlusion: A scanning electron microscope analysis. J Conserv Dent. 2017;20:269-272.

25. Zandim DL, Corrêa FO, Sampaio JE, Rossa Júnior C .The influence of vinegars on exposure of dentinal tubules: a SEM evaluation. Braz Oral Res. 2004;18:63-68.

26. Hill R, Gillam D, Karpukhina N. "Tubular occluding properties of a novel biomimetic hydroxyapatite tooth paste" in Proceedings of the IADR / PER Pan European Region
Meeting, Poster presentation (Abstract no. b 40) Helsinki, Finland, Sept 2012.

27. Goldstein J.I. et al. Coating and Conductivity Techniques for SEM and Microanalysis. In: Scanning Electron Microscopy and X-Ray Microanalysis. Springer; 1992: pp. 671-740.

28. Addy M. Dentine hypersensitivity: new perspectives on an old problem. Int Dent J. 2002;52(Suppl):367-375.

29. Zhang S. Fabrication of novel biomaterials through molecular self-assembly. Nat Biotechnol. 2003;21:1171-1178.

30. Veis A, Dorvee JR. Biomineralization mechanisms: a new paradigm for crystal nucleation in organic matrices. Calcif Tissue Int. 2013;93:307-315.

31. George A, Veis A. Phosphorylated proteins and control over apatite nulcleation, crystal growth, and inhibition. Chem Rev. 2008;108:4670-4693.

32. Yuan P, Shen X, Liu J, Hou Y, Zhu M, Huang J, et al. Effects of dentifrice containing hydroxyapatite on dentinal tubule occlusion and aqueous hexavalent chromium cations sorption: a preliminary study. PLoS One 2012;7:e45283.

33. Ishikawa K, Suge T, Yoshiyama M, Kawasaki A, Ashoka $\mathrm{K}$, Ebisu S. Occlusion of dentinal tubules with calcium phosphate using acidic calcium phosphate solution followed by neutralization. J Dent Res. 1994;73:1197-1204.

34. Hill RG, Chen X, Gillam DG. In vitro ability of a novel nanohydroxyapatite oral rinse to occlude dentine tubules. Int J Dent. 2015;2015: 153284.

35. James JM, Puranik MP, Sowmya KR. Dentinal Tubule Occluding Effect of Potassium Nitrate in Varied Forms, Frequencies and Duration: An In vitro SEM Analysis. . J Clin Diagn Res. 2017;11:ZC06-ZC08.

36. Morris MF, Davis RD, Richardson BW. Clinical efficacy of two dentin desensitizing agents. Am J Dent. 1999;12:72-76.

37. B Low S, Allen EP, Kontogiorgos ED. Reduction in dental hypersensitivity with nano-hydroxyapatite, potassium nitrate, sodium monoflurophosphate and antioxidants. Open Dent J. 2015;(9):92-97.

38. George G, Ranjini MA, Pai VS, Darsan J, Nadig RR. Evaluation of dentinal tubule occlusion using a desensitizing toothpaste and mouthwash for a period of 60 days: Scanning electron microscopy analysis. J Interdiscip Dentistry 2018; 8:96-101.

39. Nayak DS, Shetti NP. Electrochemical oxidation of provitamin B5, d-panthenol and its analysis in spiked human urine. J Anal Sci Technol. 2016;7:12. https://doi.org/10.1186/ s40543-016-0092-7. 